\title{
0-Sum and 1-Sum Flows in Regular Graphs
}

\author{
S. Akbari \\ Department of Mathematical Sciences \\ Sharif University of Technology \\ Tehran, Iran \\ School of Mathematics, \\ Institute for Research in Fundamental Sciences (IPM), \\ P.O. Box 19395-5746, \\ Tehran, Iran \\ s_akbari@sharif .edu \\ M. Kano \\ S. Zare \\ Ibaraki University \\ Hitachi, Ibaraki, Japan \\ mikio.kano.math@vc.ibaraki.ac.jp
Amirkabir University of Technology
Tehran, Iran \\ Department of Mathematical Science \\ sa_zare_f@yahoo.com
}

Submitted: Jan 20, 2015; Accepted: May 9, 2016; Published: May 27, 2016

Mathematics Subject Classifications: 05C21, 05C22, 05C70

\begin{abstract}
Let $G$ be a graph. Assume that $l$ and $k$ are two natural numbers. An $l$-sum flow on a graph $G$ is an assignment of non-zero real numbers to the edges of $G$ such that for every vertex $v$ of $G$ the sum of values of all edges incident with $v$ equals $l$. An $l$-sum $k$-flow is an $l$-sum flow with values from the set $\{ \pm 1, \ldots, \pm(k-1)\}$. Recently, it was proved that for every $r, r \geqslant 3, r \neq 5$, every $r$-regular graph admits a 0 -sum 5 -flow. In this paper we settle a conjecture by showing that every 5-regular graph admits a 0 -sum 5 -flow. Moreover, we prove that every $r$-regular graph of even order admits a 1 -sum 5 -flow.
\end{abstract}

Keywords: 0-sum flow, regular graph, 1-sum flow, factor

\section{Introduction}

Throughout this paper a graph means a finite undirected graph without loop or multiple edges. Let $G$ be a multigraph with the vertex set $V(G)$ and the edge set $E(G)$. The 
number of vertices and the number of edges of $G$ are called the order and the size of $G$, respectively. A $k$-regular graph is a graph where each vertex is of degree $k$. The degree of vertex $v$ in $G$ is denoted by $d_{G}(v)$ and $N_{G}(v)$ denotes the set of all vertices adjacent to $v$. A graph $G$ is called $k$-edge connected if the minimum number of edges whose removal would disconnect the graph is at least $k$. A pendant edge is an edge incident with a vertex of degree 1.

For a set $\left\{a_{1}, \ldots, a_{r}\right\}$ of non-negative integers an $\left\{a_{1}, \ldots, a_{r}\right\}$-graph is a graph each of whose vertices has degree from the set $\left\{a_{1}, \ldots, a_{r}\right\}$. For integers $a$ and $b, 1 \leqslant a \leqslant b$, an $[a, b]$-graph is defined to be a graph $G$ such that for every $v \in V(G), a \leqslant d_{G}(v) \leqslant b$. An $[a, b]$-factor is a spanning subgraph of $G$ in which the degree of each vertex is in the interval $[a, b]$. When $a=b$, we call it an $a$-factor.

Assume that $l$ and $k$ are two natural numbers. An $l$-sum flow on a graph $G$ is an assignment of non-zero real numbers to each edge of $G$ such that for every vertex $v$ in $V(G)$ the sum of values of all edges incident with $v$ equals $l$ and call it $l$-sum rule. An $l$-sum $k$-flow is an $l$-sum flow with values from the set $\{ \pm 1, \ldots, \pm(k-1)\}$.

Let $G$ be a graph. A $k$-flow of $G$ is an assignment of integers with maximum value at most $k-1$ to each edge of $G$ together with its orientation (or direction) such that for each vertex of $G$, the sum of the labels of incoming edges is equal to that of the labels of outgoing edges. A nowhere-zero $k$-flow is a $k$-flow with no zeros.

Tutte proposed the following interesting conjecture.

Conjecture A. (Tutte's 5-flow Conjecture [8]) If $G$ is 2-edge connected, then it has a nowhere-zero 5-flow.

In [2], it was proved that Tutte's 5-flow Conjecture is equivalent to show that every 2-edge connected bipartite graph admits a 0-sum 5-flow. In 2009, an analagous version of Tutte's Conjecture proposed for undirected graphs.

Conjecture B. (0-Sum Conjecture (ZSC) [2]) If a graph $G$ admits a 0-sum flow, then $G$ admits a 0 -sum 6-flow.

For $r$-regular graphs it was conjectured that 6 can be reduced to 5 .

Conjecture C. [1] Every $r$-regular graph $(r \geqslant 3)$ admits a 0 -sum 5-flow.

Conjecture $\mathrm{C}$ has been settled for cubic graphs in [2] and for every positive integer $r$, $r \neq 5$ in [3]. In [10], the authors proved that every $r$-regular graph $(r \geqslant 3)$ admits a 0 -sum 7 -flow. Also in [9], for some $r, k, l$, the existence of $l$-sum $k$-flow for $r$-regular graphs has been studied.

In the present manuscript using strong tools in factorization of graphs, we show that Conjecture $\mathrm{C}$ holds in general. Also, we prove that every $r$-regular graph of even order admits a 1-sum 5-flow. 


\section{0-sum 5-flow for 5-regular graphs}

The main goal of this section is showing that Conjecture $\mathrm{C}$ is true. We would like to prove the next result which settles Conjecture C.

Theorem 1. Every 5-regular graph admits a 0-sum 5-flow.

Proof. First let us state five lemmas.

Lemma 2. ([5, p.91] and [6, p.203]) Let $G$ be an n-edge connected multigraph $(n \geqslant 1), \theta$ be a real number such that $0<\theta<1$ and $f: V(G) \rightarrow\{0,1,2, \ldots\}$. If $(i)$, (ii) and one of (iiia), (iiib) hold, then $G$ has an $f$-factor.

(i) $\sum_{x \in V(G)} f(x)$ is even.

(ii) $\sum_{x \in V(G)}\left|f(x)-\theta d_{G}(x)\right|<2$.

(iiia) $n \theta \geqslant 1$ and $n(1-\theta) \geqslant 1$.

(iiib) The set $\{f(x)\}$ consists of even numbers and $m(1-\theta) \geqslant 1$, where $m \in\{n, n+1\}$ and $m \equiv 1(\bmod 2)$.

Now, we prove the following lemma.

Lemma 3. Let $G$ be a 2-edge connected $[2,5]$-multigraph. If

$3\left|\left\{x \in V(G): d_{G}(x)=2\right\}\right|+2\left|\left\{x \in V(G): d_{G}(x)=3\right\}\right|+\left|\left\{x \in V(G): d_{G}(x)=4\right\}\right| \leqslant 4$, then $G$ has a 2-factor.

Proof. Define a function $f$ on $V(G)$ as $f(x)=2$, for all $x \in V(G)$, and let $\theta=\frac{2}{5}$. Then

$$
\begin{aligned}
& \sum_{x \in V(G)}\left|f(x)-\theta d_{G}(x)\right| \\
= & \frac{6}{5}\left|\left\{x: d_{G}(x)=2\right\}\right|+\frac{4}{5}\left|\left\{x: d_{G}(x)=3\right\}\right|+\frac{2}{5}\left|\left\{x: d_{G}(x)=4\right\}\right| \\
\leqslant & \frac{8}{5}<2 .
\end{aligned}
$$

Hence Parts $(i),(i i)$ and (iiib) of Lemma 2 are satisfied with $m=3$, and thus $G$ has a 2 -factor.

In [2] the following result was proved.

Lemma 4. If $G$ is a connected $\{1,3\}$-graph and the subgraph of $G$ induced by vertices of degree 3 is 2-edge connected, then there is a function $f$ on $E(G)$ with $f(e) \in\{-2,1,4\}$ so that the 0 -sum rule holds for each vertex of degree 3 , and each pendant edge e has $f(e) \in\{-2,4\}$. Moreover, one pendant edge e may have its value pre-assigned. 
The next lemma shows that Lemma 4 can be generalized to every $\{1,3\}$-graph.

Lemma 5. Let $G$ be a connected $\{1,3\}$-graph and let $h$ be a pendant edge of $G$. For any arbitrary $\alpha \in\{-2,4\}$, there exists a function $f: E(G) \rightarrow\{-2,1,4\}$ such that $f(h)=\alpha$ and 0 -sum rule holds in each vertex of degree 3 and the value of any pendant edge is in the set $\{-2,4\}$.

Proof. Consider a rooted tree $T$ obtained from $G$ such that every maximal 2-edge connected subgraph of $G$ is considered as a vertex of $T$ and $E(T)$ consists of all cut edges of $G$, where the root is the maximal 2-edge connected subgraph one of whose vertices incident with the given pendant edge $h$, and a subgraph with one vertex is considered as a 2-edge connected subgraph. Now, we start by a root of $T$. If the root consists of one vertex, then we can easily assign the desired values to the three edges. So, we may assume that the maximal 2-edge connected subgraph, say $H$, of $G$ corresponding to the root of $T$ has order at least 2. Thus the subgraph of $G$ obtained from $H$ by adding all cut edges of $G$ incident with $H$ is a graph that satisfies the conditions of Lemma 4. Then apply Lemma 4 to obtain an edge assignment $f$ for the root with values form $\{-2,1,4\}$ in which the pendant edges have even value and $f(h)=\alpha$. Consider a maximal 2-edge connected subgraph $K$ of $G$ corresponding to a child of the root of $T$ and apply again Lemma 4 , where the edge joining $K$ to the root corresponds the given pendant edge in Lemma 4. By continuing this procedure we can find the desired function on the edge set of $G$.

Lemma 6. If $G$ is a connected $\{1,5\}$-graph, $\left\{e_{1}, \ldots, e_{s}\right\} \subseteq E(G)$ is the set of all pendant edges of $G$ and $G-\left\{e_{1}, \ldots, e_{s}\right\}$ is 2-edge connected, then there is a function $f$ on $E(G)$ with $f(e) \in\{ \pm 1, \pm 2, \pm 3, \pm 4\}$ so that 0 -sum rule holds for each vertex of degree 5 and for $i=1, \ldots, s, f\left(e_{i}\right) \in\{-2,2,4\}$. Moreover, one pendant edge $e_{l}$ may have value preassigned.

Proof. Let $s=5 p+q$, where $p \geqslant 0$ and $0 \leqslant q \leqslant 4$ are integers. We divide $5 p$ pendant edges of $G$ into $p$ groups each of which contains 5 edges, and identify the end points of every group to obtain the new $p$ vertices of degree 5 , called $v_{1}, \ldots, v_{p}$. Remove $q$ remaining pendant edges from $G$ and call the resultant multigraph by $H$. Since $H$ is obtained from $G$ by removing $q$ pendant edges, $H$ has the following property:

$$
3\left|\left\{x: d_{H}(x)=2\right\}\right|+2\left|\left\{x: d_{H}(x)=3\right\}\right|+\left|\left\{x: d_{H}(x)=4\right\}\right| \leqslant 4 .
$$

Thus by Lemma $3, H$ has a 2-factor $F$. Now, we define a function $f: E(G) \rightarrow$ $\{-2,2,3,4\}$ for $G$ so that the 0 -sum rule holds for each vertex of degree 5 . Assign value 3 to all edges of $F$, and assign value -2 to all remaining edges of $H$. Also assign value -2 to $q$ removed pendant edges of $G$. Now, if a cycle $C$ of $F$ contains at least one vertex in $\left\{v_{1}, \ldots, v_{p}\right\}$, choose one vertex, say $v_{t}$, and change the values of edges of this cycle alternatively by 2 and 4 starting at an edge incident with $v_{t}$ and ending at the other edge incident with $v_{t}$. Note that if a cycle $C$ contains no vertex in $\left\{v_{1}, \ldots, v_{p}\right\}$, do not change 
the values of edges of $C$. Then we split $5 p$ edges incident with $\left\{v_{1}, \ldots, v_{p}\right\}$ of $H$ into $5 p$ pendant edges of $G$. Thus we obtain a function $f$ with the desired property.

For the last part of lemma we consider 3 cases:

(i) $f\left(e_{\ell}\right)=-2$. Consider the graph $H$ as before. We know that $H$ contains a 2-factor $F$. If $e_{\ell}$ is not contained in $F$, then the previous assignment works. If $e_{\ell}$ is contained in $F$ and $e_{\ell}$ is incident with $v_{r}$, assign the value -3 to each edge of $F$, and assign 2 to all other edges. Then change the values of edges of the cycle $C$ alternatively by -2 and -4 , starting at $e_{\ell}$. Note that we do the same procedure for every cycle of $F$ containing a vertex in $\left\{v_{1}, \ldots, v_{p}\right\}$.

(ii) $f\left(e_{\ell}\right)=2$. If $e_{\ell}$ is contained in a 2-factor $F$ of $H$, then the previous assignment works. If $e_{\ell}$ is not contained in $F$, then assign -3 to all edges of $F$ and assign 2 to all remaining edges, and do the same procedure for every cycle $F$ containing at least one vertex in $\left\{v_{1}, \ldots, v_{p}\right\}$.

(iii) $f\left(e_{\ell}\right)=4$. Consider the first assignment of edges of $H$. If $e_{\ell}$ is contained in $F$, then we are done. If $e_{\ell}$ is not contained in 2-factor $F$ of $H$, then by removing all edges of $F$ from $H$, we obtain a $[0,3]$-graph which is not necessary connected. We have two possibilities: $e_{\ell}$ is an edge of $H-F$ or $e_{\ell}$ is not in $H$, i.e., $e_{\ell}$ is a removed pendant edge when $H$ is obtained. In the first case suppose that $v_{t}$ is a vertex of degree 3 in $H-F$ incident with $e_{\ell}$. Now, for every $v_{i} \in\left\{v_{1}, \ldots, v_{p}\right\}$, we split 3 edges of $H-F$ incident with $v_{i}$ to make 3 pendant edges. Add $q$ removed pendant edges of $G$ to $H-F$. Then the resultant graph is a $\{1,3\}$-graph, say $K$, in which $e_{\ell}$ is a pendant edge.

By Lemma 5, we have a function $g: E(K) \rightarrow\{-2,1,4\}$ such that $g\left(e_{\ell}\right)=-2$ and the values of every pendant edge is in the set $\{-2,4\}$ and moreover the 0 -sum rule holds in each vertex of degree 3 . Now, subtract 2 from all values of $E(K)$ and then multiply -1 to the values of all edges of $K$. Then assign -3 to all edges of $F$, and change the values of all edges of every cycle of $F$ containing a vertex in $\left\{v_{1}, \ldots, v_{p}\right\}$ alternatively by -2 and -4 . Clearly, the value of $e_{\ell}$ is 4 and 0 -sum rule holds for each vertex of degree 5 , as desired.

If $e_{\ell}$ is not in $H$, we add $q$ removed pendant edges of $G$ including $e_{\ell}$ to $H-F$ to obtain a $\{1,3\}$-graph, say $K$. Now, a similar method given above completes the proof.

Now, we are in a position to prove Theorem 1.

If $G$ is 2-edge connected, then by Lemma 3, $G$ has a 2 -factor $F$. Then assign value 3 to all the edges of $F$, and assign value -2 to all remaining edges of $G$, which is the desired 0 -sum 5-flow. Hence we may assume that $G$ is not 2-edge connected. Consider a rooted tree $T$ obtained from $G$ such that every maximal 2-edge connected subgraph of $G$ is considered as a vertex of $T$ and $E(T)$ consists of all cut edges of $G$, where a subgraph consisting of one vertex is considered as a 2-edge connected subgraph. Now, we start by a root of $T$ whose induced subgraph on the vertices of degree 5 is 2-edge connected. Let $H$ be the maximal 2-edge connected subgraph of $G$ corresponding to the root of $T$. Apply Lemma 6 to the subgraph of $G$ obtained from $H$ by adding all the cut edges of $G$ incident with $H$ to obtain an edge assignment for the root with values from the set $\{ \pm 1, \pm 2, \pm 3, \pm 4\}$ in which every pendant edge has a value from the set $\{-2,2,4\}$. Consider a maximal 2-edge connected subgraph $K$ of $G$ corresponding to a child of the 
root of $T$ and apply again Lemma 6 to the subgraph of $G$ obtained from $K$ by adding all cut edges of $G$ incident with $K$ to obtain an assignment on the root and $K$. By continuing this procedure we find a 0 -sum 5 -flow for $G$ and the proof is complete.

\section{1-sum flows in regular graphs}

As we mentioned that before every $r$-regular graph $r \geqslant 3$, admits a 0 -sum 5 -flow. In this section we prove that every $r$-regular graph of even order $r \geqslant 3$, admits a 1 -sum 5-flow. Before establishing our results we need some theorems.

Remark 1. We note that if a graph $G$ admits a 1 -sum $k$-flow, then $G$ has even order. To see this assume that $f$ is a 1 -sum $k$-flow for $G$. We have

$$
|V(G)|=\sum_{v \in V(G)} \sum_{u \in N_{G}(v)} f(u v)=2 \sum_{e \in E(G)} f(e) .
$$

Thus $|V(G)|$ should be even.

In the sequel we need the following result.

Theorem 7. [6 and 7, p. 184-190] Let $r \geqslant 3$ be an odd integer and let $k$ be an integer such that $1 \leqslant k \leqslant \frac{2 r}{3}$. Then every $r$-regular graph has a $[k-1, k]$-factor each component of which is regular.

Also, we need the following theorem due to Petersen.

Theorem 8. [7] Every 2k-regular multigraph admits a 2-factorization.

The following remark shows that there are some regular graphs with no 1-sum 3-flow.

Remark 2. It is not hard to see that following 3-regular graph does not admit a 1-sum 3-flow.

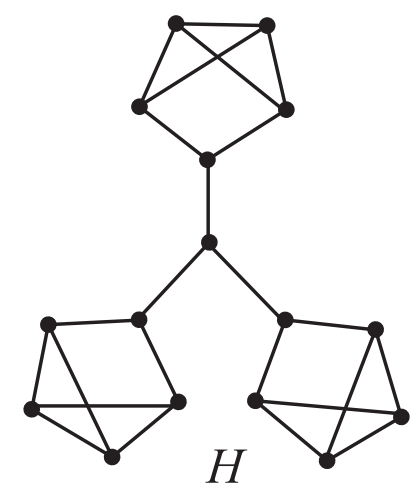

Now, we are ready to show that every $r$-regular graph of even order admits a 1-sum 5 -flow. 
Theorem 9. Let $G$ be an r-regular connected graph of even order. Then the following hold:

(i) If $r$ is an odd integer or $r=4 k+2$, for some integer $k \geqslant 0$, then $G$ admits a 1 -sum 4-flow.

(ii) If $r=4 k$, for some integer $k \geqslant 1$, then $G$ admits a 1 -sum 5 -flow.

Proof. Assume that $V(G)=\{1, \ldots, n\}$. First suppose that $r$ is an odd integer. We define a bipartite graph from $G$, called $B$, with two parts $X=\left\{x_{1}, \ldots, x_{n}\right\}$ and $Y=$ $\left\{y_{1}, \ldots, y_{n}\right\}$ and $x_{i} y_{j} \in E(B)$ if and only if $i j \in E(G)$ for every $i$ and $j, 1 \leqslant i, j \leqslant n$. So, $B$ is an $r$-regular graph and by Theorem [4, p.79], $B$ has a 1 -factorization $F_{1}, \ldots, F_{r}$. Now, for every $e \in E\left(F_{i}\right), 1 \leqslant i \leqslant r$, define a function $g: E(B) \rightarrow\left\{ \pm \frac{1}{2}, \pm \frac{3}{2}\right\}$ as follows.

For $r=4 k+1$ define:

$$
g(e)= \begin{cases}\frac{-3}{2}, & 1 \leqslant i \leqslant k \\ \frac{1}{2}, & k<i \leqslant r .\end{cases}
$$

Also, for $r=4 k+3$ define:

$$
g(e)= \begin{cases}\frac{3}{2}, & 1 \leqslant i \leqslant k+1 \\ \frac{-1}{2}, & k+1<i \leqslant r .\end{cases}
$$

Clearly, for each $u \in V(B), \sum_{v \in N_{B}(u)} g(u v)=\frac{1}{2}$. Now, define a function $f: E(G) \rightarrow$ $\{ \pm 1, \pm 3\}$ such that for every $i j \in E(G), f(i j)=g\left(x_{i} y_{j}\right)+g\left(x_{j} y_{i}\right)$. Then for every $i \in V(G), \sum_{j \in N_{G}(i)} f(i j)=1$, as desired.

Now, suppose that $r$ is an even integer. If $G$ is a 2-regular graph, then by assigning the integers $-1,2$ to the edges of $G$ alternatively, we are done.

Let $r=4 k$. Double all edges of $G$ to obtain an $8 k$-regular multigraph $G^{\prime}$. Since $G^{\prime}$ contains two edge disjoint spanning subgraphs $H_{1}$ and $H_{2}$ isomorphic to $G$ and $H_{1}$ is decomposed into 2 -factors $F_{1}, \ldots, F_{2 k}$, we can obtain a $(4 k+2)$-regular multigraph $G^{\prime \prime}=G^{\prime} \backslash E\left(F_{1}\right) \cup \cdots \cup E\left(F_{2 k-1}\right)$, which contains a $4 k$-regular graph $H_{2}$. Since $G^{\prime \prime}$ is 2-edge connected, then by Parts $(i),($ ii $)$ and (iiia) of Lemma 2, if we define $f(i)=2 k+1$, for all $i \in V\left(G^{\prime \prime}\right)$ and $\theta=\frac{1}{2}, G^{\prime \prime}$ is decomposed into two $(2 k+1)$-factors $G_{1}^{\prime \prime}$ and $G_{2}^{\prime \prime}$. Now, for every $e \in E\left(G^{\prime}\right)$, we define a function $g: E\left(G^{\prime}\right) \rightarrow\{-2,1,3\}$ as follows:

$$
g(e)= \begin{cases}-2, & e \in E\left(F_{1}\right) \cup \cdots \cup E\left(F_{k-2}\right) \cup E\left(G_{1}^{\prime \prime}\right) \\ 1, & e \in E\left(F_{k-1}\right) \cup E\left(F_{k}\right) \cup E\left(F_{k+1}\right) \cup E\left(G_{2}^{\prime \prime}\right) ; \\ 3, & e \in E\left(F_{k+2}\right) \cup \cdots \cup E\left(F_{2 k-1}\right) .\end{cases}
$$

Clearly, for each $i \in V\left(G^{\prime}\right), \sum_{j \in N_{G^{\prime}}(i)} g(i j)=1$. Now, define a function $f: E(G) \rightarrow$ $\{-4,-1,1,2,4\}$ such that for every $e \in E(G), f(e)=g(e)+g\left(e^{\prime}\right)$, where $e^{\prime}$ is the copy of $e$ in duplicating of this edge in $G^{\prime}$. Then for every $i \in V(G), \sum_{j \in N_{G}(i)} f(i j)=1$, as desired.

Now, assume that $r=4 k+2$ and $r \neq 6,10,14,22$. First note that every integer of the form $4 k+2$ can be written as $12 k+2,12 k+6$ or $12 k+10$, for some integer $k \geqslant 0$. 
Let $r=12 k+2$. Since $G$ is 2 -edge connected, then by Parts $(i),($ ii) and (iiia) of Lemma 2, if we define $f(i)=6 k+1$, for all $i \in V(G)$ and $\theta=\frac{1}{2}$, then $G$ has two $(6 k+1)$-factors $H_{1}$ and $H_{2}$. On the other hand, by Theorem $7, H_{2}$ has a $[4 k-1,4 k]$ factor, say $T$ whose components are regular. Let $T_{1}$ be the union of the $(4 k-1)$-regular components of $T$ and let $T_{2}$ be the union of $4 k$-regular components of $T$. Note that by Theorem $8, T_{2}$ has a 2 -factorization with 2 -factors $F_{1}, \ldots, F_{2 k}$. Now, we define a function $g: E(G) \backslash E\left(T_{1}\right) \rightarrow\{-3,-2,-1,2\}$ as follows:

$$
g(e)=\left\{\begin{array}{l}
-3, \quad e \in E\left(H_{2}\right) \backslash E(T) \\
-2, \quad e \in E\left(F_{i}\right), \quad 1 \leqslant i \leqslant k-1 ; \\
-1, \quad e \in E\left(F_{i}\right), \quad k \leqslant i \leqslant 2 k \\
2, \quad e \in E\left(H_{1}\right) .
\end{array}\right.
$$

Now, we want to assign some labels to the edges of $T_{1}$. With no loss of generality one can assume that $V\left(T_{1}\right)=\{1, \ldots, q\}$. We define a bipartite graph, call $L$, with two parts $X=\left\{x_{1}, \ldots, x_{q}\right\}$ and $Y=\left\{y_{1}, \ldots, y_{q}\right\}$ and $x_{i} y_{j} \in E(L)$ if and only if $i j \in E\left(T_{1}\right)$ for every $i$ and $j, 1 \leqslant i, j \leqslant q$. So, $L$ is a $(4 k-1)$-regular graph and by Theorem [4, p.79], $L$ has a 1 -factorization $F_{1}^{\prime}, \ldots, F_{4 k-1}^{\prime}$. Now, for every $e \in E\left(F_{i}^{\prime}\right), 1 \leqslant i \leqslant 4 k-1$, define a function $g^{\prime}: E(L) \rightarrow\left\{-\frac{1}{2},-\frac{3}{2}\right\}$ as follows:

$$
g^{\prime}(e)= \begin{cases}-\frac{3}{2}, & 1 \leqslant i \leqslant k-2 \\ -\frac{1}{2}, & k-1 \leqslant i \leqslant 4 k-1\end{cases}
$$

Clearly, for each $i \in V(L), \sum_{j \in N_{L}(i)} g^{\prime}(i j)=\frac{-6 k+5}{2}$. Now, define a function $f$ : $E(G) \rightarrow\{-3,-2,-1,2\}$ such that for every $e \in E(G) \backslash E\left(T_{1}\right), f(e)=g(e)$ and for every $e=i j \in E\left(T_{1}\right), f(e)=g^{\prime}\left(x_{i} y_{j}\right)+g^{\prime}\left(x_{j} y_{i}\right)$. Then for every $i \in V(G), \sum_{j \in N_{G}(i)} f(i j)=1$, as desired.

Now, suppose that $r=12 k+6$. Since $G$ is 2-edge connected, then by Parts $(i),(i i)$ and (iiia) of Lemma 2, if we define $f(i)=6 k+3$, for all $i \in V(G)$ and $\theta=\frac{1}{2}$, then $G$ has two $(6 k+3)$-factors $H_{1}$ and $H_{2}$. On the other hand, by Theorem $7, H_{2}$ has a $[4 k+1,4 k+2]$-factor, say $T$ whose components are regular. Let $T_{1}$ be the union of the $(4 k+1)$-regular components of $T$ and let $T_{2}$ be the union of $(4 k+2)$-regular components of $T$. Note that by Theorem $8, T_{2}$ has a 2 -factorization with 2 -factors $F_{1}, \ldots, F_{2 k+1}$. Now, we define a function $g: E(G) \backslash E\left(T_{1}\right) \rightarrow\{-3,-2,-1,2\}$ as follows:

$$
g(e)=\left\{\begin{array}{l}
-3, \quad e \in E\left(H_{2}\right) \backslash E(T) \\
-2, \quad e \in E\left(F_{i}\right), \quad 1 \leqslant i \leqslant k \\
-1, \quad e \in E\left(F_{i}\right), \quad k+1 \leqslant i \leqslant 2 k+1 \\
2, \quad e \in E\left(H_{1}\right) .
\end{array}\right.
$$

Now, we want to assign some labels to the edges of $T_{1}$. With no loss of generality one can assume that $V\left(T_{1}\right)=\{1, \ldots, q\}$. We define a bipartite graph, call $L$, with two parts $X=\left\{x_{1}, \ldots, x_{q}\right\}$ and $Y=\left\{y_{1}, \ldots, y_{q}\right\}$ and $x_{i} y_{j} \in E(L)$ if and only if $i j \in E\left(T_{1}\right)$ for every $i$ and $j, 1 \leqslant i, j \leqslant q$. So, $L$ is a $(4 k+1)$-regular graph and by Theorem [4, p.79], $L$ has a 1 -factorization $F_{1}^{\prime}, \ldots, F_{4 k+1}^{\prime}$. Now, for every $e \in E\left(F_{i}^{\prime}\right), 1 \leqslant i \leqslant 4 k+1$, define a function $g^{\prime}: E(L) \rightarrow\left\{-\frac{1}{2},-\frac{3}{2}\right\}$ as follows: 


$$
g^{\prime}(e)= \begin{cases}-\frac{3}{2}, & 1 \leqslant i \leqslant k-1 \\ -\frac{1}{2}, & k \leqslant i \leqslant 4 k+1 .\end{cases}
$$

Clearly, for each $v \in V(L), \sum_{v \in N_{L}(u)} g^{\prime}(u v)=\frac{-6 k+1}{2}$. Now, define a function $f$ : $E(G) \rightarrow\{-3,-2,-1,2\}$ such that for every $e \in E(G) \backslash E\left(T_{1}\right), f(e)=g(e)$ and for every $e=i j \in E\left(T_{1}\right), f(e)=g^{\prime}\left(x_{i} y_{j}\right)+g^{\prime}\left(x_{j} y_{i}\right)$. Then for every $i \in V(G), \sum_{j \in N_{G}(i)} f(i j)=1$, as desired.

Next, assume that $r=12 k+10$. Since $G$ is 2-edge connected, then by Parts $(i),(i i)$ and (iiia) of Lemma 2, if we define $f(i)=6 k+5$, for all $i \in V(G)$ and $\theta=\frac{1}{2}$, then $G$ has two $(6 k+5)$-factors $H_{1}$ and $H_{2}$. On the other hand, by Theorem $7, H_{2}$ has a $[4 k+1,4 k+2]$-factor, say $T$ whose components are regular. Let $T_{1}$ be the union of the $(4 k+1)$-regular components of $T$ and let $T_{2}$ be the union of $(4 k+2)$-regular components of $T$. Note that by Theorem $8, T_{2}$ has a 2 -factorization with 2 -factors $F_{1}, \ldots, F_{2 k+1}$. Now, we define a function $g: E(G) \backslash E\left(T_{1}\right) \rightarrow\{-3,-2,-1,2\}$ as follows:

$$
g(e)= \begin{cases}-3, & e \in E\left(H_{2}\right) \backslash E(T) \\ -2, & e \in E\left(F_{i}\right), \quad 1 \leqslant i \leqslant k-1 ; \\ -1, & e \in E\left(F_{i}\right), \quad k \leqslant i \leqslant 2 k+1 ; \\ 2, & e \in E\left(H_{1}\right) .\end{cases}
$$

Now, we want to assign some labels to the edges of $T_{1}$. With no loss of generality one can assume that $V\left(T_{1}\right)=\{1, \ldots, q\}$. We define a bipartite graph, call $L$, with two parts $X=\left\{x_{1}, \ldots, x_{q}\right\}$ and $Y=\left\{y_{1}, \ldots, y_{q}\right\}$ and $x_{i} y_{j} \in E(L)$ if and only if $i j \in E\left(T_{1}\right)$ for every $i$ and $j, 1 \leqslant i, j \leqslant q$. So, $L$ is a $(4 k+1)$-regular graph and by Theorem [4, p.79], $L$ has a 1 -factorization $F_{1}^{\prime}, \ldots, F_{4 k+1}^{\prime}$. Now, for every $e \in E\left(F_{i}^{\prime}\right), 1 \leqslant i \leqslant 4 k+1$, define a function $g^{\prime}: E(L) \rightarrow\left\{-\frac{1}{2},-\frac{3}{2}\right\}$ as follows:

$$
g^{\prime}(e)= \begin{cases}-\frac{3}{2}, & 1 \leqslant i \leqslant k-2 \\ -\frac{1}{2}, & k-1 \leqslant i \leqslant 4 k+1 .\end{cases}
$$

Clearly, for each $v \in V(L), \sum_{v \in N_{L}(u)} g^{\prime}(u v)=\frac{-6 k+3}{2}$. Now, define a function $f$ : $E(G) \rightarrow\{-3,-2,-1,2\}$ such that for every $e \in E(G) \backslash E\left(T_{1}\right), f(e)=g(e)$ and for every $e=i j \in E\left(T_{1}\right), f(e)=g^{\prime}\left(x_{i} y_{j}\right)+g^{\prime}\left(x_{j} y_{i}\right)$. Then for every $i \in V(G), \sum_{j \in N_{G}(i)} f(i j)=1$, as desired.

Now, suppose that $G$ is an $r$-regular graph such that $r \in\{6,10,14,22\}$ and $r=4 k+2$. Since $G$ is 2-edge connected then by Parts $(i),(i i)$ and (iiia) of Lemma 2, if we define $f(i)=2 k+1$, for all $i \in V(G)$ and $\theta=\frac{1}{2}$, then $G$ has two $(2 k+1)$-factors $G_{1}$ and $G_{2}$. Then by Theorem $7, G_{2}$ has a $[t-1, t]$-factor $T$, for every $t, 1 \leqslant t \leqslant \frac{2 r}{3}$, whose components are regular. Let $T_{1}$ be the union of the $(t-1)$-regular components of $T$ and let $T_{2}$ be the union of $t$-regular components of $T$.

If $r=6$, then $G_{2}$ has a [1,2]-factor. Define a function $f: E(G) \rightarrow\{-2,1,2,3\}$, where $f(e)=-2$ for $e \in E\left(G_{1}\right), f(e)=3$ for $e \in E\left(G_{2}\right) \backslash E(T), f(e)=1$ for $e \in E\left(T_{1}\right)$ and $f(e)=2$ for $e \in E\left(T_{2}\right)$. 
If $r=10$, then $G_{2}$ has a [1,2]-factor. Define a function $f: E(G) \rightarrow\{-2,-1,1,3\}$, where $f(e)=-2$ for $e \in E\left(G_{1}\right), f(e)=3$ for $e \in E\left(G_{2}\right) \backslash E(T), f(e)=-1$ for $e \in E\left(T_{1}\right)$ and $f(e)=1$ for $e \in E\left(T_{2}\right)$.

If $r=14$, then $G_{2}$ has a [3,4]-factor. Note that by Theorem 8, $T_{2}$ has two 2-factors, say $T_{1}^{\prime}$ and $T_{2}^{\prime}$. Now, define a function $f: E(G) \rightarrow\{-3,-1,-2,2\}$, where $f(e)=2$ for $e \in E\left(G_{1}\right), f(e)=-1$ for $e \in E\left(G_{2}\right) \backslash E(T), f(e)=-3$ for $e \in E\left(T_{1}\right), f(e)=-2$ for $e \in E\left(T_{1}^{\prime}\right)$ and $f(e)=-3$ for $e \in E\left(T_{2}^{\prime}\right)$.

If $r=22$, then $G_{2}$ has a [2,3]-factor. Define a function $f: E(G) \rightarrow\{-3,1,2,3\}$, where $f(e)=2$ for $e \in E\left(G_{1}\right), f(e)=-3$ for $e \in E\left(G_{2}\right) \backslash E(T), f(e)=3$ for $e \in E\left(T_{1}\right)$ and $f(e)=1$ for $e \in E\left(T_{2}\right)$.

Then for every $i \in V(G), \sum_{j \in N_{G}(i)} f(i j)=1$, as desired.

We close the paper with the following conjecture.

Conjecture 10. Every connected 4k-regular graph of even order admits a 1-sum 4-flow.

Acknowledgements. The authors are indebted to the Institute for Research in Fundamental Sciences (IPM) for support. The research of the first and the third authors were in part supported by a grant from IPM (No. 93050212) and (No. 9313452), respectively. The second author is supported by Grant-in-Aid for Scientific Research of Japan.

\section{References}

[1] S. Akbari, A. Daemi, O. Hatami, A. Javanmard and A. Mehrabian, Zero-sum flows in regular graphs, Graphs and Combinatorics 26 (2010) 603-615.

[2] S. Akbari, N. Gharaghani, G.B. Khosrovshahi, A. Mahmoody, On zero-sum 6-flows of graphs, LAA 430 (2009) 3047-3052.

[3] S. Akbari, N. Gharghani, G.B. Khosrovshahi, S. Zare, A note on zero-sum 5-flows in regular graphs, The Electronic Journal of Combinatorics 19(2) (2012), \#P7.

[4] J.A. Bondy and U.S.R. Murty, Graph Theory with Applications, North Holland, New York (1976).

[5] M. Kano, [a,b]-factorization of a graph, J. Graph Theory, 9 (1985) 129-146.

[6] M. Kano, Factors of regular graph, J. Combin. Theory Ser. B 41 (1986), 27-36.

[7] J. Petersen, Die Theorie der regularen Graphen. Acta Math(15) (1891), 193-220.

[8] W.T. Tutte, A contribution to the theory of chromatic polynomials, Canadian J. Math. 6, (1954). 80-91.

[9] T.-M. Wang, S.-W. Hu, Constant sum flows in regular graphs, FAW-AAIM 2011, Lecture Notes in Computer Science, Springer Verlag 6681, (2011) 168-175.

[10] T.-M. Wang, S.-W. Hu, Zero-sum flow numbers of regular graphs, FAW-AAIM 2012, Lecture Notes in Computer Science, Springer Verlag 7285, (2012) 269-278. 